\title{
Dissociating Arbitrary Stimulus-Response Mapping from Movement Planning during Preparatory Period: Evidence from Event-Related Functional Magnetic Resonance Imaging
}

\author{
Cristiana Cavina-Pratesi, ${ }^{1}$ Kenneth F. Valyear, ${ }^{1}$ Jody C. Culham, ${ }^{1}$ Stefan Köhler, ${ }^{1}$ Sukhvinder S. Obhi, ${ }^{2}$ \\ Carlo Alberto Marzi, ${ }^{3}$ and Melvyn A. Goodale ${ }^{1}$ \\ ${ }^{1}$ Department of Psychology, University of Western Ontario, London, Ontario, Canada N6A 5C2, ${ }^{2}$ Department of Psychology, Wilfrid Laurier University, \\ Waterloo, Ontario, Canada N2L 3G1, and ${ }^{3}$ Department of Neurological and Vision Sciences, University of Verona, 37134 Verona, Italy
}

In the present study, we aimed to dissociate the neural correlates of two subprocesses involved in the preparatory period in the context of arbitrary, prelearned stimulus-response (S-R) associations, namely, S-R mapping and movement planning (MP). We teased apart these two subprocesses by comparing three tasks in which the complexity of both S-R mapping and MP were independently manipulated: simple reaction time (SRT) task, go/no-go reaction time (GNGRT) task, and choice reaction time (CRT) task. We found that a more complex S-R mapping, which is the common element differentiating CRT and GNGRT from SRT, was associated with higher brain activation in the left superior parietal lobe (SPL). Conversely, a greater number of planned finger movements, which is the common difference between CRT and both SRT and GNGRT, was associated with higher brain activation in a number of frontal areas, including the left supplementary motor area (SMA), left dorsal premotor cortex (dPM), and left anterior cingulate cortex (ACC). The left-hemisphere dominance for S-R mapping could be related to the fact that arbitrary S-R mapping is often verbally mediated in humans. Overall, these results suggest a clear dissociation in the preparatory-set period between the more abstract role of left SPL in activating the appropriate S-R associations and the more concrete role played by the SMA, dPM, and ACC in preparing the required motor programs.

Key words: fMRI; frontal cortex; superior parietal lobe; motor preparation; S-R mapping; preparatory period; human

\section{Introduction}

Knowing in advance the required response to a particular stimulus can improve the speed and accuracy of performance. This improvement is thought to depend on the advance preparation of several constituent subprocesses (Jennings and van der Molen, 2005). For example, when approaching a set of traffic lights, anticipation of the rules that govern stopping and proceeding (red light, pressing the brake; green light, pressing the accelerator) and advance preparation of each possible foot movement will facilitate the appropriate action. In the domain of arbitrary stimulusresponse ( $\mathrm{S}-\mathrm{R}$ ) mapping, in which the stimulus characteristics do not automatically invoke the correct response, knowing the rules of the particular S-R contingency is fundamental to the efficient preparation of subsequent responses. An important question is whether the neural correlates of mapping S-R association and

Received July 29, 2005; revised Jan. 20, 2006; accepted Jan. 22, 2006.

This work was supported by grants from the Natural Sciences and Engineering Research Council of Canada, the Canada Research Chairs Program (M.A.G.), and the Ministero Istruzione Universitá Ricerca (C.A.M.). We are grateful to Alessandra Fanini and three anonymous reviewers for their comments on this manuscript, Paul Gribble and Joseph DeSouza for the stimulating discussions, and Matthew Brown and Christopher Thomas for their help with Matlab software analysis. We also thank Joe Gati and Joy Williams for MRI advice and data collection.

Correspondence should be addressed to Cristiana Cavina-Pratesi, Department of Psychology, University of Durham, Queen's Campus, Stockton, University Boulevard, Thornaby, Stockton-on-Tees TS17 6BH, UK. E-mail: cristiana.cavina-pratesi@durham.ac.uk.

DOI:10.1523/JNEUROSCI.3176-05.2006

Copyright $\odot 2006$ Society for Neuroscience $\quad$ 0270-6474/06/262704-10\$15.00/0 movement planning (MP) are mediated by different brain regions.

Functional neuroimaging studies in humans (Brass and von Cramon, 2002, 2004a,b; Thoenissen et al., 2002; Bunge et al., 2003) (for review, see Bunge, 2005) and neurophysiological studies in macaques (Stoet and Snyder, 2004; Pasupathy and Miller, 2005) have investigated the neural correlates of preparatory set in prelearned arbitrary S-R associations. A common finding is the involvement of a number of areas located in the frontal and parietal cortices. Although activity in both brain regions is modulated during the delay period between the presentation of instructional cues and the presentation of the stimuli, little is known about whether these different regions are selectively involved in different subprocesses of the preparatory period. The goal of the present study was to dissociate the neural substrates of S-R mapping from the neural substrates of MP during the preparatory period.

We performed an event-related functional magnetic resonance imaging (fMRI) experiment in which we examined brain activation during the preparatory period in three manual visuomotor conditional reaction time (RT) tasks: simple RT (SRT), go/no-go RT (GNGRT), and choice RT (CRT). Overall, the three tasks differ in the number and kind of subprocesses required to arrive at the final response: GNGRT and CRT (but not SRT) require stimulus discrimination, whereas only CRT (but neither GNGRT nor SRT) requires response selection. Thus, during the 


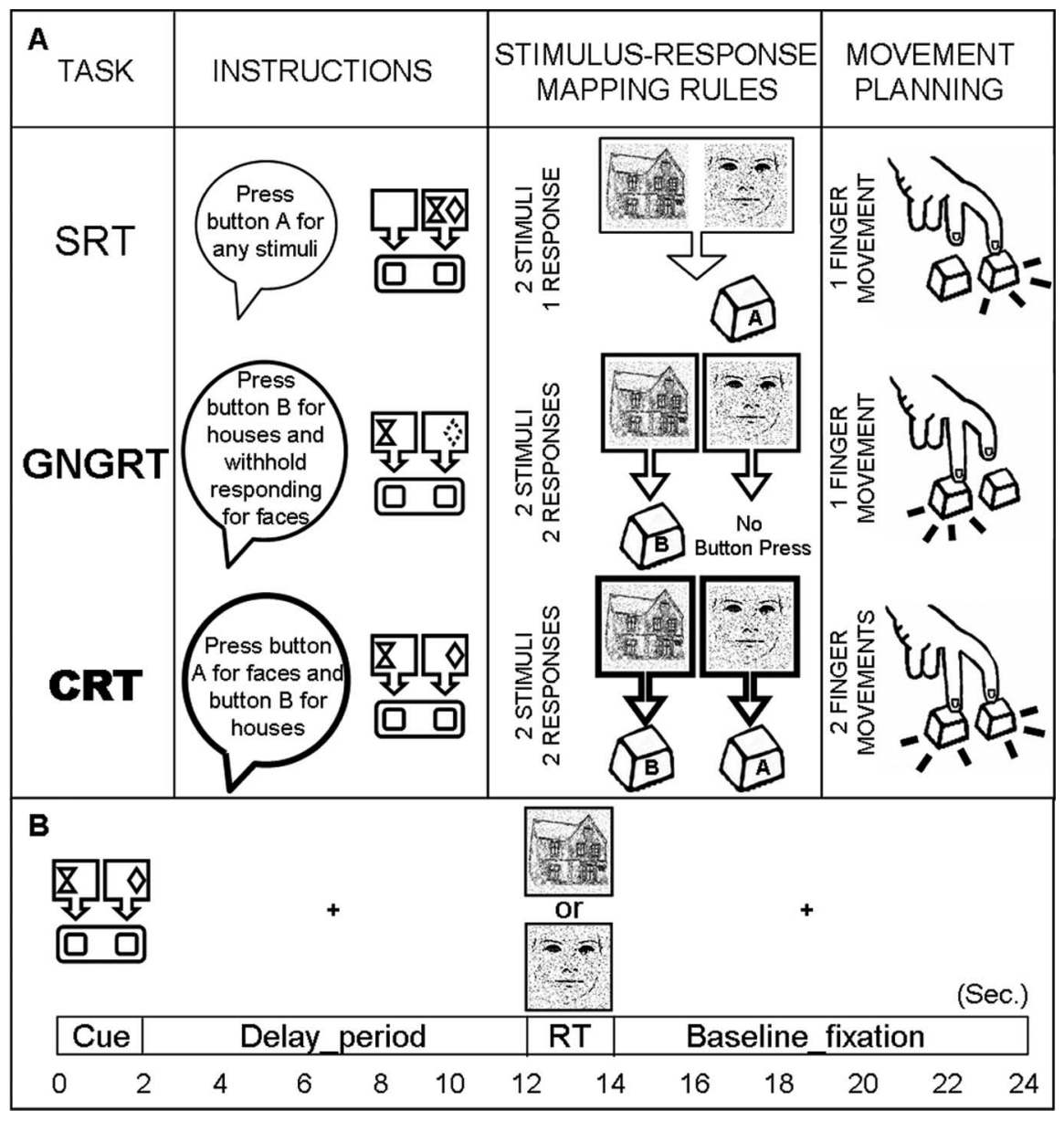

Figure 1. A, Schematic representation of the different components underling task preparation in SRT, GNGRT, and CRT. Under Instructions, the exact guidelines given to the subjects verbally before the experimental session and the pictorial instruction they saw at the beginning of each trial for the three tasks are reported. Under Stimulus-Response Mapping Rules, the links between stimulus (S) and response (R) association for each task are highlighted. Under Movement Planning, the required possible finger movements are listed. In the SRT task, subjects were required to press button A after the presentation of any visual stimulus (faces or houses) (i.e., there was only one S-R association to activate and one finger movement to prepare). In the GNGRT task, subjects were required to press button $B$ for houses and withhold responding for faces (or vice versa) (i.e., there were two $S$-R mappings to activate but still only one finger movement to prepare). Finally, in the CRT task, subjects were required to press button $A$ for faces and button $B$ for houses (or vice versa) (i.e., there were two $S-R$ mappings to activate and two finger movements to prepare). $\boldsymbol{B}$, Timing organization of a representative trial. Time is expressed in seconds (Sec.), and each event (cue, delay_period, RT, and baseline_fixation) is a multiple of the TR ( $2 \mathrm{~s}$ ). Each trial is composed of four periods that appear in sequence: instruction period (2 s), delay period (10 s), RT-task period (including stimulus onset and RT collection; $2 \mathrm{~s}$ ), and the baseline-fixation period (10 s).

preparatory period, when the upcoming task is known but the stimulus has not yet been presented, there will be differences among the tasks in the complexity of both the required S-R mappings and the MP (Fig. 1A). Although each task requires at least one S-R association to map and one finger movement to plan, GNGRT and CRT differed from SRT, because they both involved a more complex S-R association. In contrast, CRT differed from both GNGRT and SRT, because it required preparing for two possible finger movements instead of only one.

We reasoned that tasks sharing the more difficult S-R association (CRT and GNGRT) would selectively activate brain areas involved in S-R mapping, whereas the task involving a more complex movement planning (CRT) would reveal areas subserving MP.

\section{Materials and Methods}

Subjects and procedures

Ten right-handed participants (five females; mean age, 25.1 years) performed the three RT tasks in a high-field MRI system. All of the partici- pants provided informed consent before beginning the experiment, which was conducted in accordance with local ethical guidelines and conforms with The Code of Ethics of the World Medical Association (Declaration of Helsinki). Before the actual experiment, to familiarize participants with the S-R association, a separate practice session was conducted. Aside from a shorter delay period, the practice session was otherwise identical to that performed in the magnet.

Each trial lasted $24 \mathrm{~s}$ and consisted of a cue period (during which instructions were presented for $2 \mathrm{~s}$ ), a delay period (which consisted of 10 s delay-fixation interval), a task period (during which the subjects responded to a stimulus that was presented for $100 \mathrm{~ms}$ ), and a last baseline fixation period (during which a fixation cross was presented for $11.9 \mathrm{~s}$ ) (Fig. $1 B$ ).

Instruction stimuli consisted of two horizontally aligned squares (resembling the two buttons to press) on which the visual stimuli (faces and houses) were to be mapped (Fig. 1A). Two symbols (a diamond and a clepsydra) were used to represent either faces or houses and were assigned to each subject in a counterbalanced way. The rationale behind the use of symbols instead of real faces and houses as cues was to ensure that the processes underlying the preparatory period were as abstract as possible (that is without any sensory information associated with the incoming stimuli). To make the following description simpler, consider the case in which the diamond represented faces and the clepsydra represented houses (as shown in Fig. 1A). When both symbols were mapped onto the same square (on the right, for example), the subject had to press the corresponding button (on the right) for faces and houses; this was the SRT condition. When, for example, a clepsydra was mapped onto the left square and the dotted diamond was mapped onto the right square, the subject had to press the left button for houses and withhold the response for faces; this was the GNGRT condition. Note that the GNGRT condition yielded two "subconditions" depending on whether the go stimulus was a house or a face; GNGRT_house and GNGRT_face, respectively. Finally, when a diamond was mapped onto the right square, for example, and the clepsydra was mapped onto the left one, the participant had to press the right button for faces and the left button for houses; this was the CRT condition. The number of button presses for the second and the third fingers was counterbalanced for the three tasks. In the actual experiment, for each subject, during GNGRT and CRT trials, faces and houses were always mapped onto the same right or left button. Thus, although the association of the symbols with the visual stimuli and the association of the visual stimuli with the left and right button were kept constant for any particular participant, the association of the symbols with the visual stimuli was counterbalanced between participants.

After the instruction period and before the stimulus presentation, a gray fixation point $\left(0.57 \times 0.57^{\circ}\right.$ of visual angle at a distance of $\left.50 \mathrm{~cm}\right)$ was presented for $10 \mathrm{~s}$. The pictures of faces and houses $\left(8.5 \times 8.5^{\circ}\right.$ of visual angle at a distance of $50 \mathrm{~cm}$ ) were chosen from a set of colored stimuli (courtesy of Tzvika Ganel, Ben Gurion University of the Negev, Be'er Sheva, Israel) and were manipulated as follows: they were first converted to grayscale, then traced along the major contours, and finally superimposed onto a random noise background (Fig. $1 A, B$ ). Instructions, visual stimuli, and the fixation cross were presented in the center of the screen. Participants were required to respond as quickly and accu- 
rately as possible when the visual stimulus was presented using either the index finger or middle finger of the right hand. At stimulus offset, the fixation cross reappeared for $11.9 \mathrm{~s}$, and participants were required to fixate. Participants were explicitly asked not to press the buttons during the delay period and not to correct their responses in case of errors. RT and error rate were recorded on-line.

Each scanning session consisted of eight functional runs and one highresolution anatomical acquisition. Each functional run comprised 16 trials (four trials for each RT task: SRT, GNGRT_house, GNGRT_face, and CRT) and lasted $\sim 7 \mathrm{~min}$. During each run, trials were pseudorandomly ordered.

\section{Imaging}

Imaging was performed at the Robarts Research Institute (London, Ontario, Canada) using a 4 tesla whole-body Siemens-Varian (Erlangen, Germany; Palto Alto, CA) MRI system. A transmit-receive, cylindrical birdcage radiofrequency head coil was used in all experiments. Blood oxygenation level-dependent (BOLD)-based (Ogawa et al., 1992) functional MRI volumes were collected using an optimized segmented $\mathrm{T} 2{ }^{\star}$ weighted spiral acquisition scheme $(64 \times 64$ matrix size; echo time $(\mathrm{TE})$, $15 \mathrm{~ms}$; repetition time (TR), $500 \mathrm{~ms}$; flip angle (FA), $30^{\circ}$; four segments per plane, $22.0 \mathrm{~cm}$ field of view; volume acquisition time, $2 \mathrm{~s}$ ). Each volume comprised 17 contiguous pseudo-axial slices at $5 \mathrm{~mm}$ thickness. A constrained, three-dimensional phase shimming procedure was performed to optimize the magnetic field homogeneity over the prescribed functional planes (Klassen and Menon, 2004). Physiologic fluctuations were compensated for every segment of every slice using a point-based navigator correction scheme collected at the beginning of every spiral readout. Slices were acquired at a $30^{\circ}$ caudal tilt with respect to the anterior commissure to posterior commissure (ACPC) line (Damasio, 1995). For each participant, a T1-weighted anatomic reference volume was acquired along the same orientation as the functional images using a three-dimensional SPIRAL acquisition sequence $(256 \times 256 \times 64$ matrix size; $3.0 \mathrm{~mm}$ reconstructed slice thickness; inversion time, $1300 \mathrm{~ms}$; TR, $50 \mathrm{~ms}$; TE, $3.0 \mathrm{~ms} ; \mathrm{FA}, 30^{\circ}$ ).

\section{Data analysis}

Behavioral data. RTs and accuracy (error rates) were collected on-line. Errors and no-response trials were excluded from the imaging analysis. RT and accuracy were analyzed using ANOVA and two-tailed $t$ test statistics.

Imaging data. Data were analyzed using BrainVoyager 2000 software package (Brain Innovation, Maastricht, The Netherlands). For each subject, functional data from each session were screened for motion and/or magnet artifacts with cine-loop animation. No head motion artifacts were observed, probably because our subjects were highly experienced, and therefore no motion correction was applied (Freire and Mangin, 2001). Functional data were superimposed on anatomical brain images, aligned on the ACPC line, and transformed into Talairach space (Talairach and Tournoux, 1988). Functional data were preprocessed with linear trend removal and spatial smoothing (full-width half-maximum, 6 $\mathrm{mm}$ ). Matlab version 6.1 (MathWorks, Natick, MA) was used to remove all error trials from the functional data files. Data were then analyzed using a fixed-effect general linear model with separate predictors for each phase in each task condition. Because there were no behavioral differences between responses to houses versus faces (see Results), we averaged each task across faces and houses, resulting in 10 different phases: three phases for the instructions (SRT, GNGRT, and CRT), three phases for the delay period (SRT, GNGRT, and CRT), and four phases for the task [which includes the stimulus presentation and the subject's response (SRT, GNGRT, CRT, and withhold-GNGRT for no-go trials)]. The final period of fixation at the end of each trial (10 s) was used as a baseline. For each task, sustained activity during the delay was modeled as an epoch with its onset time locked to the offset of the instructions and with duration matched to the length of the delay (10 s; i.e., five volumes). The instruction period and the task period (stimulus onset plus button press or withheld button press) were both modeled as a transient ( 2 s; i.e., one volume) epoch. The square wave function for each phase was convolved with a canonical hemodynamic response function (Boynton et al., 1996).

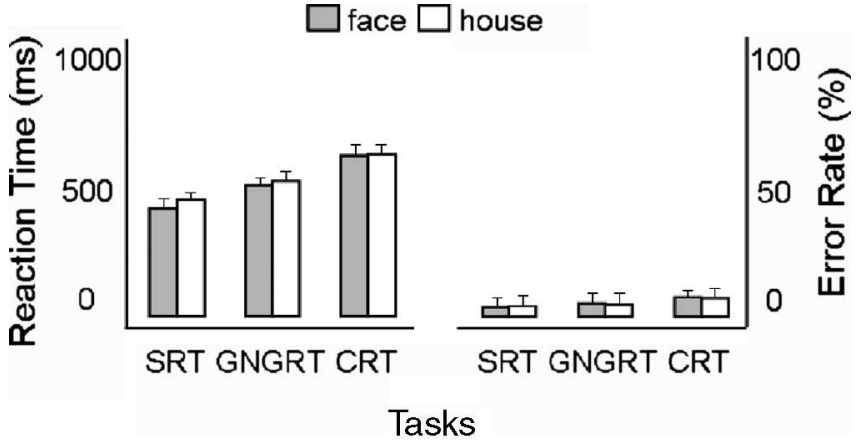

Figure 2. Behavioral results. The reaction time (left) and error rate (right) as a function of the stimulus and task are shown. Error bars indicate SE.

Statistical activation maps were set to reliable threshold levels and cluster volumes $\left(p<0.001\right.$; minimum cluster size, $\left.162 \mathrm{~mm}^{3}\right)$ using Monte Carlo simulations (performed with AlphaSim software; courtesy of Douglas Ward, Medical College of Wisconsin, Milwaukee, WI) to verify that our regions of interest were unlikely to have arisen because of chance given the problem of multiple comparisons.

After having identified the areas that were activated by a comparison of interest, we performed post hoc analyses on the event-related time course as follows. We extracted the event-related time course for each subject and each task condition separately. We then calculated the percentage of BOLD signal change (\%BSC) for the delay period by averaging data points from 2 to $10 \mathrm{~s}$ (see time course in Figs. 3,4) and by comparing task conditions using two-tailed $t$ test statistics with subject-related variability as error estimates.

\section{Results}

\section{Behavioral data}

Behavioral results in terms of reaction time and accuracy are shown in Figure 2. Inspection of the raw RT data revealed that there were no excessively fast or excessively slow button presses (i.e., there were no RTs $\geq 3$ SDs from the mean). The number of errors (and so the number of discarded trials) was measured by a two-way repeated-measures ANOVA using TASK (SRT, GNGRT, and CRT) and STIMULI (faces and houses) as withinsubjects factors. None of the factors were significant, demonstrating that errors were equally distributed among the tasks (5\% for SRT, 7\% for GNGRT, and $8 \%$ for CRT) and the stimulus type (6\% for houses and $7 \%$ for faces). We also performed a two-way repeated-measures ANOVA on the mean RT using TASKS and STIMULI as within-subject factors. The TASK factor was found to be significant $\left(F_{(2,20)}=54.6 ; p=0.0001\right)$, and a post hoc $t$ test showed that subjects were significantly faster in the SRT condition (RT, $415.7 \mathrm{~ms}$ ) compared with both the GNGRT condition (RT, $498.1 \mathrm{~ms}$ ) and the CRT condition (RT, $604.6 \mathrm{~ms}$ ), which were significantly different from each other (all of the comparisons were significant at the $p<0.0001$ level). These results are in agreement with previous findings demonstrating that differences among the three RT tasks might be related to the gradual increase of the number of cognitive processes from SRT to GNGRT and from GNGRT to CRT tasks (Donders, 1969). Thus, the high accuracy and the significant differences in RT across the three tasks imply that the subjects were carefully following the instructions presented at the beginning of each trial by preparing the three tasks in different ways.

\section{Imaging data}

Paired-comparisons between tasks

The behavioral results clearly show that progressively more time is required to perform the three tasks. Which brain areas are 
Table 1. Areas significantly active for the comparison of GNGRT > SRT, CRT > GNGRT, and CRT > SRT

\begin{tabular}{|c|c|c|c|c|c|c|c|}
\hline \multirow[b]{2}{*}{ Contrasts } & \multirow{2}{*}{$\begin{array}{l}\text { Brain } \\
\text { areas }\end{array}$} & \multirow{2}{*}{$\begin{array}{c}\text { Brodmann } \\
\text { area }\end{array}$} & \multicolumn{3}{|c|}{ Talairach coordinates } & \multirow{2}{*}{$\begin{array}{c}\text { Volume } \\
\left(\mathrm{mm}^{3}\right)\end{array}$} & \multirow{2}{*}{$\begin{array}{c}\text { \%BSC } \\
\text { ( } t \text { test, } \\
p \text { value) }\end{array}$} \\
\hline & & & $x$ & $y$ & $Z$ & & \\
\hline GNGRT $>$ SRT & Left SPL & BA7 & -29 & -50 & 44 & 592 & 0.014 \\
\hline \multirow[t]{3}{*}{$\mathrm{CRT}>\mathrm{GNGRT}$} & Left dPM & BA6 & -30 & -8 & 52 & 421 & 0.018 \\
\hline & Left SMA & BA6 & -8 & -2 & 59 & 209 & 0.017 \\
\hline & Left ACC & BA32/23 & -14 & 18 & 42 & 282 & 0.009 \\
\hline \multirow[t]{4}{*}{ CRT $>$ SRT } & Left SPL & BA7 & -29 & -50 & 45 & 683 & 0.007 \\
\hline & Left dPM & BA6 & -28 & -8 & 52 & 574 & 0.008 \\
\hline & Left SMA & BA6 & -8 & 3 & 63 & 209 & 0.002 \\
\hline & Left ACC & BA32/23 & -12 & 16 & 40 & 291 & 0.003 \\
\hline
\end{tabular}

For each area, Talairach coordinates, volume in $\mathrm{mm}^{3}$, Brodmann area, and statistical significance for \% $\mathrm{BSC} t$ test comparisons are reported.

$$
\text { - SRT }=\text { GNGRT }- \text { CRT }
$$
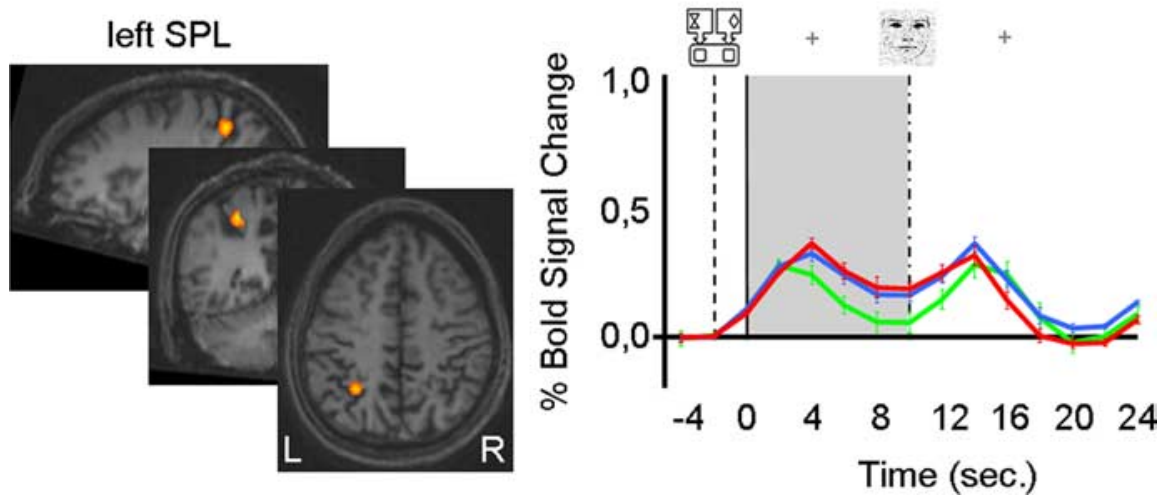

Figure 3. Group activation map and \%BSC for the conjunction analysis involving the delay period: [(CRT vs SRT) and (GNGRT vs SRT)]. Only one area in the left SPL (BA7; Talairach coordinates, $x=-29, y=-50, z=43$; volume, $534 \mathrm{~mm}^{3}$ ) was more active for GNGRT and CRT compared with SRT. Two-tailed post hoc $t$ tests showed that the \%BSC for GNGRT did not differ from that for CRT $\left(t_{(9)}=-0.927 ; p=0.378\right)$, and the \%BSC for both was significantly higher than SRT (GNGRT vs SRT, $t_{(9)}=3.98, p=0.003 ;$ (CRT vs $\left.S R T, t_{(9)}=5.45 ; p=0.0001\right)$. The $\% B S($ for the delay period was calculated by averaging data points in a time window between 2 and $10 \mathrm{~s}$. Data are based on Talairach-averaged group results, shown for clarity on a single subject's anatomical area (which is not representative of sulcal patterns for all subjects). The gray area highlights the length of the delay period. The dotted line represents instructions onset; the solid line represents delay period onset; the dotted and pointed line represents stimulus onset; sec. represents seconds. Error bars indicate SE.

recruited during the preparation period as the tasks became progressively more complex? To answer this question, we compared each task with each of the less complex ones as follows: GNGRT $>$ SRT, CRT $>$ GNGRT, and CRT $>$ SRT. The contrast of GNGRT > SRT revealed activation in the left superior parietal lobe (SPL), particularly in the left horizontal section of the intraparietal sulcus (IPS) (Duvernoy, 1999), located in Brodmann area (BA) 7. The stereotaxic coordinates for the activated area and the $p$ value for the \%BSC are shown in Table 1 . In a second comparison, we contrasted CRT $>$ GNGRT and found activations in three frontal foci: the left supplementary motor area (SMA) in the left medial wall of BA 6; the left dorsal premotor cortex (dPM) in the precentral gyrus (BA 6); and the left anterior cingulate sulcus (ACC) in the medial wall BA 32 (Table 1). When we compared CRT $>$ SRT, we localized activations in all of the foci mentioned in the previous two analyses, that is, in the left parietal and frontal cortices: the left SPL, the left SMA, the left $\mathrm{dPM}$, and the left ACC (Table 1). From the above results, it seems that as more and more cognitive processes are about to be engaged (requiring more and more processing time) an increasing level of activation is evident in a number of areas along the frontoparietal network during the preparatory phase. Tasks that differ only in the complexity of S-R mapping (GNGRT > SRT) show a corresponding difference in the level of activation in the left SPL (but not in frontal areas), whereas tasks that differ only in the complexity of MP (CRT > GNGRT) show a corresponding difference in the level of activation in frontal areas (but not in parietal areas). Finally, tasks that differ in the complexity of both S-R mapping and $\mathrm{MP}(\mathrm{CRT}>\mathrm{SRT})$ show differences in the level of activation in both parietal and frontal areas.

\section{Conjunction analysis: $S-R$ mapping and $M P$}

To isolate the neural correlates of S-R mapping from the neural correlates of $\mathrm{MP}$ across the three tasks, we performed a conjunction analysis. Although standard subtraction logic can be usefully applied to experiments involving two tasks that differ in one or more cognitive components (Donders, 1969), when more than two tasks are involved, it is more useful to use a conjunction analysis that can identify components that are shared between some tasks but not others. In conjunction analysis, multiple comparisons are adjoined with an "and" function. To illustrate the utility of this approach, consider an fMRI experiment with three tasks: A, B, and C. If we identify voxels that exhibit a common activation in a particular brain region for both tasks $\mathrm{A}$ and $\mathrm{B}$ compared with task $\mathrm{C}$, we can use a conjunction analysis adjoining the contrast $\mathrm{A}>\mathrm{C}$ with the contrast $\mathrm{B}>\mathrm{C}$. The resultant activation map would "flag" voxels as significant only if they were to satisfy both of these arguments. As such, conjunction analyses can provide more rigorous evidence with respect to the neural substrates of the presumptive cognitive process than single contrasts alone (Price and Friston, 1997). Note that this is not the same procedure as simply grouping $\mathrm{A}$ and $\mathrm{B}$ together and contrasting this against condition $\mathrm{C}$ (i.e., $[(\mathrm{A}+\mathrm{B})-\mathrm{C}])$ ). This comparison would flag both of those voxels that are higher for $\mathrm{A}$ compared with $\mathrm{C}$ and those voxels that are higher for $\mathrm{B}$ compared with C. It would not select only those voxels that are common to both contrasts (i.e., it would not be as conservative and rigorous as a conjunction analysis). Because our three tasks differed from each other in terms of S-R mapping and/or MP component, by using the conjunction approach, we were able to localize clusters of activity selectively associated with S-R mapping only and MP only.

Both the GNGRT and CRT require mapping different stimuli onto different responses, whereas the SRT does not. More specifically, GNGRT differs from SRT only for the S-R mapping required, whereas CRT differs from SRT with respect to the S-R mapping required as well as the number of possible finger movements to prepare. To identify brain regions related to S-R mapping only, we performed a conjunction analysis between the two pairs of conditions that differed in this component (GNGRT vs SRT and CRT vs SRT). This conjunction analysis revealed activation in the left SPL, in exactly the same location identified previously with the paired-comparison analysis. The activated area and the \%BSC are shown in Figure 3.

The CRT requires the preparation of two possible finger 
movements, whereas the GNGRT and SRT tasks do not. More specifically, CRT differs from GNGRT only in terms of the number of possible finger movements to prepare, whereas CRT differs from SRT with respect to the S-R mapping required as well as the number of possible finger movements to prepare. To identify brain regions involved in MP only, we performed a conjunction analysis between the two task pairs that differed in this component (CRT vs GNGRT and CRT vs SRT). This conjunction analysis revealed activations in three different foci of the left frontal cortex: the left SMA, the left dPM, and the left ACC. The activated areas and the $\%$ BSC are shown in Figure 4.

\section{Single-subject data}

To ensure that the activations detected in the group analysis were also reflected in the individual subjects' data, we examined the activation maps for each subject individually. Embracing a purely descriptive approach, we searched for peaks of activity in individual brains ( $p<0.05$ uncorrected) around the peak voxels found in the group analysis. When CRT $>$ SRT was combined with GNGRT $>$ SRT in a conjunction analysis, the majority of the subjects showed activation around the left IPS in the SPL ( 8 of 10 subjects; averaged Talairach coordinates: $x=-35, y=-51$, $z=46$; SD: $x=3.8, y=4.7, z=3.7)$. A closer inspection revealed that the activations were most often located in the medial wall of the IPS. When the second conjunction analysis [(CRT $>$ SRT $)$ and (CRT > GNGRT)] was applied, activations for the majority of the subjects were found around the left dPM ( 8 of 10; averaged Talairach coordinates: $x=-31, y=$ $-8, z=52$; SD: $x=6.0, y=4.3, z=4.8$ ), the left SMA (9 of 10; averaged Talairach coordinates: $x=-9, y=-1, z=60$; SD: $x=3.7, y=1.8, z=2.2)$, and the left ACC (8 of 10; averaged Talairach coordinates: $x=-7, y=16, z=41$; SD: $x=3.3, y=$ $6.1, z=5$ ). These results indicate that our findings at the group level are indeed consistent across individual subjects.

\section{Ruling out differences related to top- down control of visual attention}

In SRT, there is no need to discriminate among the stimuli, because every stimulus requires the same response. In the GNGRT and CRT tasks, however, stimulus discrimination is a requirement for the correct response execution. Thus, it is likely that when the stimuli were present, more attentional resources were deployed for the GNGRT and CRT tasks than for the SRT task. However, it is less clear that such attentional deployment would be invoked during the delay period. Nevertheless, it is important to rule out the possibility that such anticipatory deployment of
SRT $=$ GNGRT - CRT
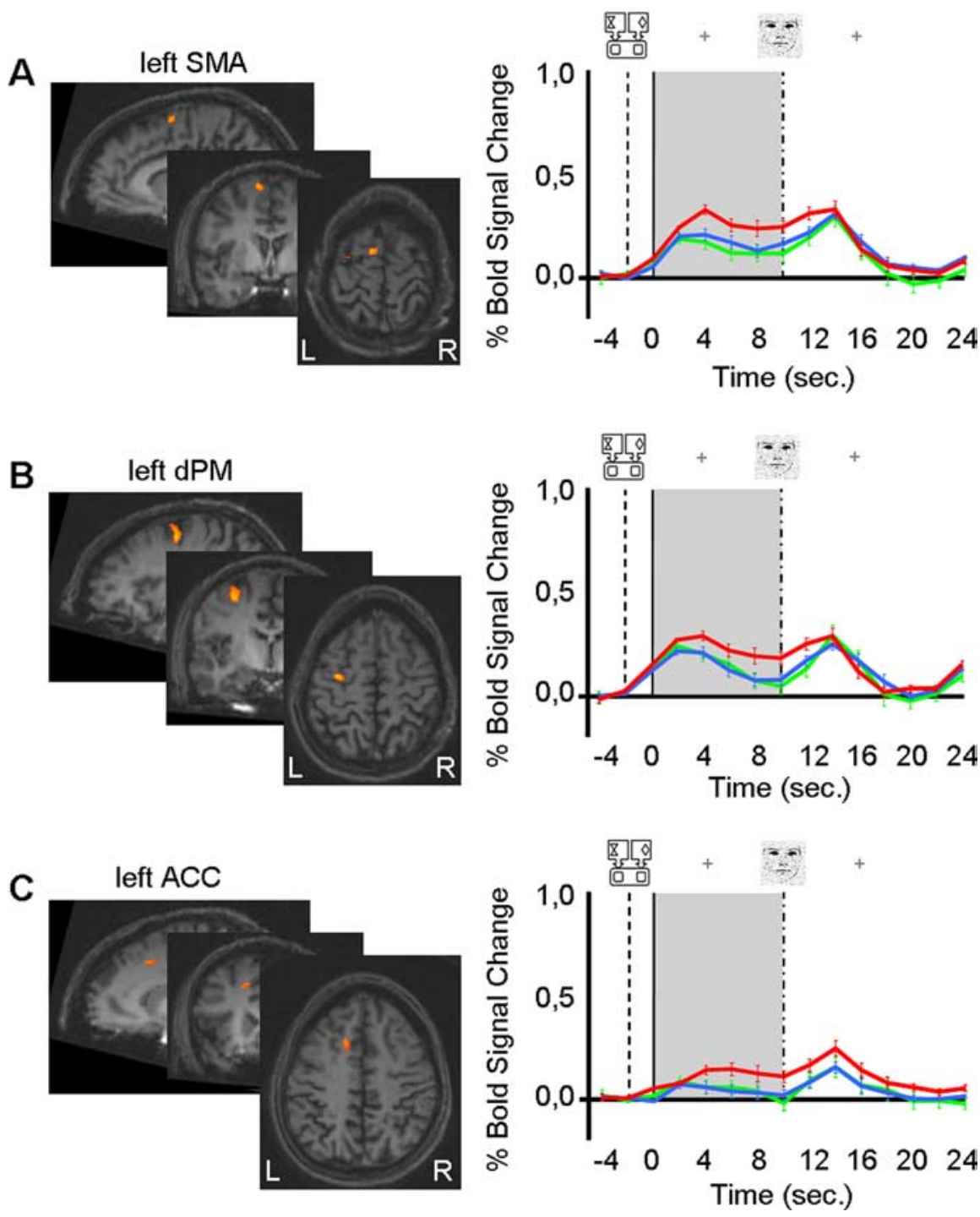

Figure 4. Group activation maps and \%BSC for the conjunction analysis involving the delay period [(CRT vs SRT) and (CRT vs GNGRT)]. Three areas showed greater activation for (RT compared with both GNGRT and SRT: $A$, left SMA (BA6; Talairach coordinates, $x=-9 ; y=-2, z=60$; volume, $248 \mathrm{~mm}^{3}$ ); $B$, left dPM (BA6; Talairach coordinates, $x=-30 ; y=-8, z=51$; volume, $686 \mathrm{~mm}^{3}$ ); and $\boldsymbol{C}$, left ACC (BA 32/23; Talairach coordinates, $x=-13, y=15, z=42 ;$ volume, $234 \mathrm{~mm}^{3}$ ). Two-tailed post hoc t tests showed that in the left SMA, \%BSC was higher for the delay period in CRT than for the delay period in GNGRT or SRT (CRT vs SRT, $t_{(9)}=7.21, p=0.001$; (RT vs GNGRT, $\left.t_{(9)}=4.76, p=0.001\right)$. In addition, SRT and GNGRT did not differ from one another. Similarly, in the left dPM, \%BSC was higher for CRT compared with GNGRT $\left(t_{(9)}=6.7 ; p=0.0001\right)$ or SRT $\left(S R T, 0.11 ; t_{(9)}\right.$ $=3.7 ; p=0.005)$, which did not differ from one another. Finally, in the left ACC, \%BSC was significantly greater for CRT than for $\operatorname{SRT}\left(t_{(9)}=7.2 ; p=0.001\right) \operatorname{or~GNGRT~}\left(t_{(9)}=5.3 ; p=0.001\right)$, which again did not differ from one another. The \%BSC for the delay period was calculated by averaging data points in a time window between 2 and $10 \mathrm{~s}$. Data are based on Talairach-averaged group results shown for clarity on a single subject's anatomical area (which is not representative of sulcal patterns for all subjects). The gray area highlights the length of the delay period. The dotted line represents instructions onset; the solid line represents delay period onset; the dotted and pointed line represents stimulus onset; sec. represents seconds. Error bars indicate SE. attention could explain the higher activation in the SPL for the GNGRT and CRT tasks. There is evidence, after all, that cognitive expectations can sometimes guide attention to select in advance some characteristics of the upcoming stimuli such as their spatial location or specific object features. This type of attentional modulation is commonly referred to as "top-down control of attention" (for review, see Corbetta and Shulman, 2002). The network of areas generally activated when the top-down control of attention is directed to stimulus features includes parietal, frontal, and extrastriate cortices (Corbetta and Shulman, 2002). If the present 


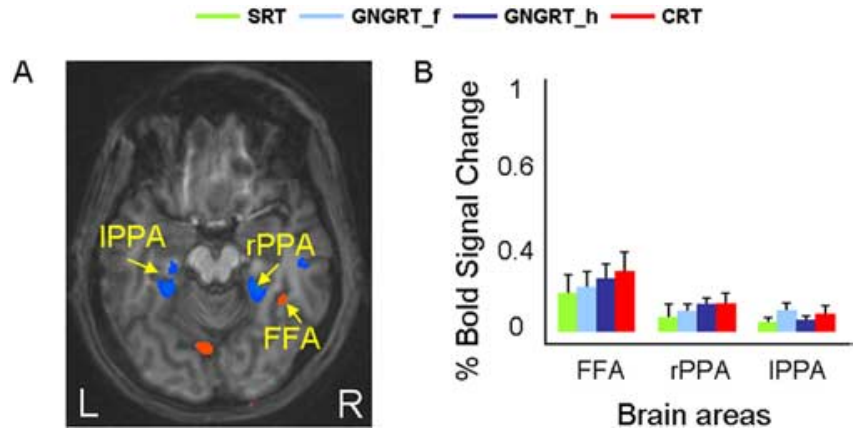

Figure 5. Task modulation in FFA and PPA during the delay period. $A$, Brain areas activated by comparing faces (SRT_f,GNGRT_f, and CRT_f) versus houses (SRT_h, GNGRT_h, and CRT_h) during the task period. FFA (Talairach coordinates, $x=37, y=-41, z=-15$ ) is shown in orange, and PPA (Talairach coordinates: right, $x=23, y=-39, z=-11$; left, $x=-27$, $y=-40, z=-7$ ) is shown in blue. $B, \%$ BSC for SRT, GNGRT_f, GNGRT_h, and (RT trials during the delay period as a function of PPA and FFA. The \%BSC for the delay period was calculated by averaging data points in a time window between 2 and 10 s. Error bars indicate SE.

activation found in the SPL was related to the top-down control of visual attention to discriminate faces from houses, then one would expect increased activity in the visual areas devoted to face and house recognition, namely fusiform face area (FFA) and parahippocampal place area (PPA) for CRT and GNGRT compared with SRT. Such attentional top-down effects have been demonstrated clearly in previous fMRI studies (Kastner et al., 1999). However, our conjunction analysis (i.e., [(CRT > SRT) and $($ GNGRT $>$ SRT) $]$ ) did not reveal any activation in FFA and PPA. To confirm that the absence of activation was not related to a lack of statistical power, we performed an additional analysis in which we first localized FFA and PPA by comparing face trials versus house trials during the task period. For this purpose, we split our trials into SRT_f, SRT_h, GNGRT_f, GNGRT_h, CRT_f and CRT_h. The comparison of face versus house [(SRT_f, GNGRT_f, CRT_f) > (SRT_h, GNGRT_h, CRT_h)] showed unilateral right activation for FFA and bilateral activation for PPA (Fig. 5A). We then checked whether the \%BSC in FFA and PPA during the delay period was modulated differently for GNGRT and CRT compared with SRT as one would expect if topdown control of attention was engaged. Analysis of the BOLD signal was performed by splitting GNGRT trials into GNGRT for house (i.e., when subjects were required to press the button for houses only; GNGRT_h) and GNGRT for faces (i.e., when subjects were required to press the button for faces only; GNGRT_f). A post hoc $t$ test showed that there was no difference in the \% BSC for SRT, GNGRT_h, GNGRT_f, and CRT in FFA (for all contrasts, $p>0.47$ ) and PPA (for all contrasts: left, $p>0.9$; right, $p>$ 1) (Fig. $5 B$ ). This pattern of results suggests that our subjects did not use any more top-down control of attention in the delay periods of the GNGRT and CRT tasks than they did in the delay period of the SRT task. In fact, the two tasks were extremely simple in terms of the required visual discriminations. Not only are faces and houses easy to tell apart, but unlike other studies in which the stimuli to be discriminated were presented together, a face or house was always presented on its own. After all, when the target was finally presented, there would have been no need to bring to bear elaborated attentional mechanisms such as target selection and distractor inhibition, processes which might have otherwise been ramped up in anticipation of their required deployment (Wojciulik et al., 1998; Beck and Kastner, 2005).

An attentional-load argument (Wojciulik and Kanwisher, 1999; Culham et al., 2001) can also be ruled out given that the change in activation in the SPL in our study does not map onto the observed change in RT performance across the three tasks, namely a gradual increase in RTs from SRT to GNGRT to CRT.

\section{Ruling out differences related to working memory}

It is possible that the maintenance of information in working memory (WM) during the long delay period could account for some of the brain activations we described. Getting ready to perform the task during the delay and keeping the task instructions in memory is a fine distinction that has been highlighted previously in the preparatory-period literature (Toni et al., 2001). In contrast to response preparation, WM requires the conscious act of keeping information in mind for short periods of time (within the scale of seconds). According to the most widely accepted WM model (for review, see Baddeley, 2003), such a conscious act of short-term maintenance is mediated by the "phonological loop" or by the "visuospatial sketchpad" depending on the nature of the material to be maintained. In this scenario, the higher activation found for CRT and GNGRT compared with SRT might be associated with subvocal rehearsal involved in maintaining the instructions on-line in the more complex tasks. In other words, while during SRT the subject might have waited passively for the stimulus to appear, in CRT and GNGRT, they might have rehearsed silently the more complex S-R associations. However, we think that such an interpretation cannot account for our results.

First of all, previous studies investigating the neural correlates of the phonological loop have identified the left inferior parietal lobe (BA 40) as the locus of the storage component of the loop, and Broca's area 6/44 as the primary region involved in the rehearsal component (Smith and Jonides, 1997). The pattern of activation that we found in the present study does not fit with this picture, because our parietal activation was localized in the superior, instead of the inferior, parietal lobe and because there was no activation in Broca's area. Similarly, we ruled out a visuospatial scratchpad explanation, in that we observed no selective righthemisphere activation (Baddeley, 2003).

To obtain even stronger evidence against the WM interpretation, we also conducted a simple behavioral experiment in which we tested the likelihood that subjects recruited the phonological loop during the delay period with an articulatory suppression paradigm (Baddeley et al., 1984). Subjects $(n=8)$ were asked to perform two sessions of the same task performed during scanning: in one session, they performed the task in the same way as they did in the magnet, but in a second session, they were required to count backwards in steps of two from a given number during the delay period. If the higher activation for the more complex tasks observed in the fMRI study was related to the maintenance of information in WM, then the suppression of subvocal rehearsal by counting should selectively affect performance only in those tasks (namely GNGRT and CRT). We ran two different two-way repeated-measure ANOVAs on RT and error rate using SESSION (subvocal rehearsal and no subvocal rehearsal) and TASK (SRT, GNGRT, and CRT) as factors. For the reaction time data, we observed that the counting task, which should have suppressed subvocal rehearsal, did indeed slow down performance $\left(F_{(1,7)}=14.7 ; p=0.004\right)$, but it affected all tasks in the same way (Fig. 6). In other words, although the three tasks differed in $\operatorname{RT}\left(F_{(2,14)}=52.6 ; p=0.0001\right)$, there was no interaction with $\operatorname{SESSION~}\left(F_{(2,14)}=0.285 ; p=0.76\right)$. As shown in Figure 6, error rate was not affected by the counting task $\left(F_{(1,7)}=0.041 ; p=0.844\right)$ and was the same across the three tasks $\left(F_{(2,14)}=0.104 ; p=0.902\right)$. 
$\square$ sub-vocal rehearsal $\square$ no sub-vocal rehearsal

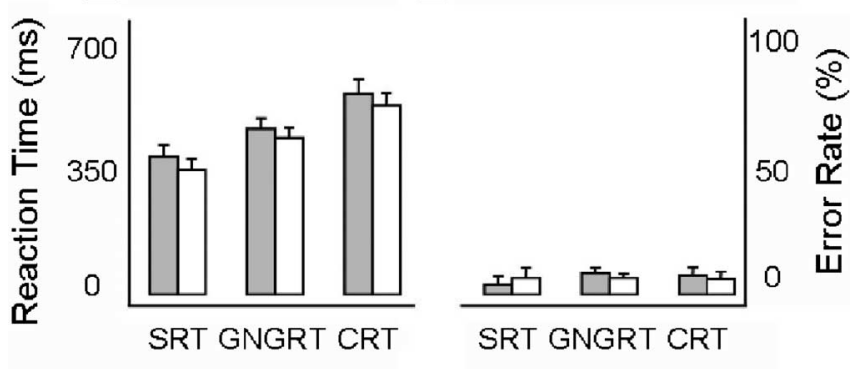

Tasks

Figure 6. Working memory. The reaction time (left) and error rate (right) for subvocal rehearsal (gray) and no subvocal rehearsal (white) trials as a function of tasks are shown. Error bars indicate $S E$.

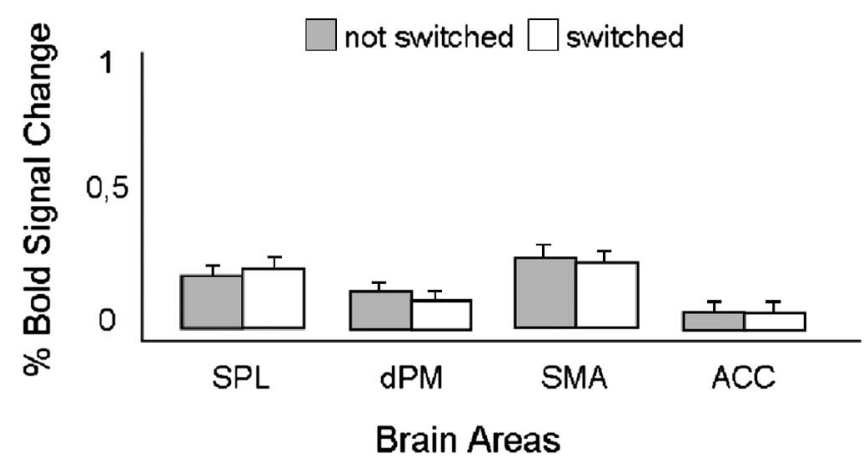

Figure 7. Task switching. The \%BSC for switched (in white) and not switched (in gray) trials as a function of the investigated areas is shown. Error bars indicate SE.

\section{Ruling out differences related to task switching}

Because our subjects were asked to switch between the three tasks in a random manner, it is possible that the differences in brain activation observed might be related to the cognitive cost associated with switching from one task to another (for review, see Allport et al., 1994). In the preparatory-set imaging literature, task-switching paradigms have been used to study rule reconfiguration mostly in prefrontal cortex (Kimberg et al., 2000; Sohn et al., 2000; Luks et al., 2002; Brass and von Cramon, 2004b; Brass et al., 2005; Bunge, 2005; Crone et al., 2005). It could be the case that the activations found in the left SPL and in the left frontal regions were associated with switching to a more difficult task in terms of S-R mapping (switching from SRT to GNGRT or CRT) or motor output (switching from SRT or GNGRT to CRT), respectively. To rule out this possibility, we divided our trials into switched (S) and notswitched (NS) trials. We extracted each subject's eventrelated time course for S and NS trials from the areas found by our main comparisons, and we analyzed the \%BSC. In all areas (left SPL, left dPM, left SMA, and left ACC), there was no difference $(p>0.5)$ between $S$ and NS trials (Fig. 7). The lack of task-switching activity in the SPL and the frontal areas is easily explained by taking into account the fact that in our paradigm, in contrast to previous studies, subjects switched between tasks (i.e., the nature of the required response, pressing or inhibiting) but not between rules (the specific link between buttons and stimuli). With the exception of SRT, which required a minimal amount of S-R mapping (and showed the lower activation in all the investigated brain areas), faces and houses were always mapped onto the same button within a particular subject.

\section{Ruling out differences related to instruction retrieval}

To rule out the possibility that our results were related to differences in retrieving the instructions presented just before the delay period, we performed the same conjunction analyses on the instruction period ( $2 \mathrm{~s}$ at the beginning of each trial). No significant activation was found at the given threshold, a result that suggests that common rather than different brain areas were involved in instruction retrieval in each of the three RT tasks. This lack of differential activation does not seem to be attributable to insufficient statistical power; when we averaged across tasks to identify regions that were common to rule retrieval, the activated areas were found to overlap with those found in previous studies (Thoenissen et al., 2002; Bunge et al., 2003). Specifically, cue period activations were observed in a wide range of cortical areas, including bilateral primary and secondary visual areas (BA 17/ 18 ), precuneus (BA 19), superior and inferior parietal lobe (BA $40 / 7$ ), superior temporal cortex (BA 22), posterior cingulate cortices (BA 23), frontal cortices located in the superior, and medial frontal gyrus (BA 6 and 8), together with prefrontal cortices (BA 9). In addition, subcortical areas were also active and included bilateral regions of the putamen and thalamus.

\section{Discussion}

In the present study, we dissociated the neural correlates of two subprocesses involved in preparatory set, namely S-R mapping and MP. We teased apart these two subprocesses by manipulating the complexity of S-R associations and the number of possible finger movements across three manual RT tasks requiring increasingly complex cognitive operations: SRT, GNGRT, and CRT. We found that a more complex S-R mapping was associated with activation in the left SPL. In contrast, we found that a larger number of planned finger movements was associated with left frontal activation in the SMA, dPM, and ACC. Moreover, additional analyses of the neuroimaging data and a behavioral follow-up experiment allowed us to rule out alternate interpretations of the results in terms of object attention, working memory, task switching, or instruction retrieval.

A clear functional distinction between the different components of preparatory set and their anatomical correlates in the frontparietal network for the domain of arbitrary S-R mapping has not been shown previously. Previous studies have provided only inconsistent results (Toni et al., 2001; Thoenissen et al., 2002; Bunge et al., 2003; Brass and von Cramon, 2004b). One possible explanation is that S-R mapping and MP in these studies have been confounded (Bunge et al., 2003; Brass and von Cramon, 2004b) or studied separately (Toni et al., 2001; Thoenissen et al., 2002). The present study was able to reveal a straightforward distinction between parietal and frontal activations, because the experimental design involved three separate tasks in which the complexity of both S-R mapping and MP were manipulated independently.

\section{Parietal lobe and S-R mapping}

Our finding of an involvement of parietal cortex in S-R mapping is consistent with a number of studies that have investigated response selection in manual RT experiments. S-R mapping is a subprocess of a more general cognitive mechanism called response selection, which has been found to depend on activity in a network involving frontal and parietal areas (Hazeltine et al., 2000; Bunge et al., 2002; Jiang and Kanwisher, 2003a,b; Schumacher et al., 2003). In all studies, activity related to S-R mapping was not distinguishable from activity related to another subprocess of response selection, namely inhibition of inappropriate 
responses. Although the differential involvement of the parietal and frontal cortices in S-R mapping and response inhibition, respectively, has been suggested in some of these studies, the distinction was not directly investigated (Bunge et al., 2000; Hazeltine et al., 2003; Jiang and Kanwisher, 2003a,b). The present results, which were based on an analysis of the delay period, support the idea that the parietal lobe is selectively involved in mapping S-R associations; although anticipation of the possible S-R associations can occur during the delay period before the stimulus is presented, any inhibition of inappropriate or preponderant responses can only be exerted after the presentation of the stimulus.

Our demonstration of parietal involvement in S-R mapping is also in agreement with previous studies investigating the neural correlates of preparatory set (Bunge et al., 2003; Brass and von Cramon, 2004a,b). In these experiments, parietal activation increased as a direct result of increasing the number of S-R alternatives. For example, the parietal activation for CRT was higher than SRT (Bunge et al., 2003), the activation for preparing two different S-R mapping tasks was larger than for preparing only one task (Brass and von Cramon, 2004a), and the activation for incongruent trials (which also evokes competing S-R mappings) was higher compared with neutral trials (Brass and von Cramon, 2004b).

An important point to address is the nature of the sustained S-R mapping activity found in the SPL. We already discarded explanations related to processes such as keeping the S-R association in memory, switching between S-R associations, or attending to the more demanding task in terms of visual discrimination. One more possibility is that the sustained activity reflects a topdown control of attention specifically related to "assembling" stimuli and responses (Corbetta and Shulman, 2002). It is likely that during the delay period of an arbitrary S-R mapping task, attention is needed to assemble stimuli and responses that are unrelated and therefore not automatically linked (Corbetta and Shulman, 2002). Previous studies have observed activations in the vicinity of the left IPS that are related to altering the S-R mapping either by changing the required motor output (Rushworth et al., 2001) or the relevant stimulus dimension (Shulman et al., 2002). It is possible (although the current literature is not clear on this point) that the concept of attentional selection for S-R mapping (Corbetta and Shulman, 2002) may not be that different from the concept of "motor attention" (Boussaoud and Wise, 1993a,b; Rushworth et al., 2003). Motor attention has been defined as the "covert intentional processes that are related to overt limb movements" (Rushworth et al., 2001), and it is possible that the specific process of mapping a stimulus onto a response is part of such a covert intentional process. In other words, the covert intention to move one limb and not the other (motor attention) in an arbitrary RT task is strongly related to the correct application of the S-R contingency.

The left-hemisphere dominance for S-R mapping found in the present study has been reported previously (Corbetta and Shulman, 2002), and it has been linked to the idea that arbitrary S-R mapping is typically verbally mediated in humans (Shulman et al., 2002). This account might also explain why the large removal of the SPL in macaque did not affect performance in arbitrary S-R mapping tasks (Rushworth et al., 1997). Thus, there may be a real species difference here: humans learn arbitrary S-R associations by using verbal mediation, whereas monkeys use operative motor learning (Passingham, 1993).

Finally, another possible explanation of the activity in SPL should be acknowledged. Perhaps the activity related to the wellknown role of this region in spatial/visuomotor transformations is being suppressed to implement the more arbitrary S-R association. There are certainly some examples in the neuroimaging literature of areas where changes in BOLD activity related to suppression have been described. For example, there is the so-called "default mode network," which appears to be suppressed when participants are performing a task (vs simply resting). But the suppression of activity in this network of "association areas," which includes posterior cingulate, inferior parietal, and medial prefrontal cortex, is associated not with an increase in the BOLD signal (our observation) but rather with a decrease (Raichle, 2001; Greicius et al., 2003; Greicius and Menon, 2004; Fox et al., 2005). In any case, the suppression account seems unlikely given that the increased activity in the SPL was observed during the delay period before any stimulus (that might have invoked a response) was even present.

\section{Frontal areas and MP}

The general involvement of frontal areas in MP is widely accepted (Picard and Strick, 2001), and preparatory-period activity for finger movements has been reported in a variety of RT imaging experiments (Deiber et al., 1996; Tanji, 1996; Petit et al., 1998; Lee et al., 1999; Toni et al., 1999, 2001; Thickbroom et al., 2000; Pochon et al., 2001; Brass and von Cramon, 2002; Thoenissen et al., 2002; Cunnington et al., 2003; Huang et al., 2004).

More specifically, our finding of greater activation in the $\mathrm{APM}$ cortex when subjects prepared two finger movements (CRT) as opposed to one (GNGRT and SRT) is in agreement with previous studies that have observed modulation of premotor neurons in movement selection during arbitrary S-R association tasks (Wise et al., 1992; Wise, 1993). Moreover, TMS studies showed that stimulation over the APM slows down RT for CRT but not SRT tasks when applied either during the movement (Schluter et al., 1998) or during the preparatory-set phase (Schluter et al., 1999). It is unlikely that differences in response probability or response possibility, which have also been found for preparatory-set activations in the dPM (Toni et al., 2001; Thoenissen et al., 2002; Cisek and Kalaska, 2005), could explain the results obtained by our second conjunction analysis. Response probability would predict that the pattern of activation in the APM should be the same for CRT and SRT (in which the probabilities of executing a movement are $100 \%$ ), and this pattern should be quite different from that seen with GNGRT (in which the probability of executing a movement is only 50\%). Conversely, response possibility would predict a higher pattern of activation in the dPM for CRT and GNGRT (in which there are two response options, pressing button A vs button B for CRT and pressing vs withholding the response for GNGRT) compared with SRT (in which there is only one response option). However, this is not what we observed.

The present activation in the ACC is consistent with other findings that have correlated activity in this area with monitoring and adjustment of performance when conflicting or competitive responses are required (Botvinick et al., 2004). Of course, during the preparatory period, when the response has not yet been executed, the role of ACC cannot be in adjusting for possible errors that have not yet been made; instead, it is probably more concerned with monitoring. That the activation for CRT, in which two competing movements were being prepared, was higher than the activation for GNGRT, in which two competing responses were being prepared (response execution or response inhibition), suggests that the role of ACC may be more related to monitoring between conflicting movements rather than between conflicting responses. Nevertheless, this idea of a more "motoric" role for the $\mathrm{ACC}$ is not in disagreement with recent reviews proposing that 
the ACC subserves both cognitive and motor functions (Paus, 2001; Picard and Strick, 2001).

Finally, the activation we observed in the SMA can be interpreted in a straightforward manner as reflecting the complexity of the motor demands, in that the CRT condition, which showed the highest activation in this region, required two possible movements, whereas only one movement was required in the SRT and GNGRT conditions (Roland et al., 1980).

\section{Conclusions}

We dissociated two subprocesses of preparatory set in the context of arbitrary, prelearned S-R associations, namely stimulusresponse mapping and MP. We found that whereas the left SPL was involved in mapping S-R contingency, the left $\mathrm{dPM}$, left SMA, and left ACC were involved in the preparation of the actual motor output. In short, we demonstrated a clear dissociation in the preparatory period between the more abstract role of the left SPL in preparing the possible response options and the more concrete role played by the SMA, dPM, and ACC in alerting the required motor programs.

\section{References}

Allport A, Styles E, Hsieh S (1994) Shifting intentional set: exploring the dynamic control of tasks. In: Attention and performance XV: conscious and nonconscious information processing (Umilta C, Moscovitch M, eds). Hillsdale, NJ: Erlbaum.

Baddeley A (2003) Working memory: looking back and looking forward. Nat Rev Neurosci 4:829-839.

Baddeley A, Lewos V, Vallar G (1984) Exploring the articulatory loop. Q J Exp Psychol A 36:223-252.

Beck DM, Kastner S (2005) Stimulus context modulates competition in human extrastriate cortex 8:1110-1116.

Botvinick MM, Cohen JD, Carter CS (2004) Conflict monitoring and anterior cingulate cortex: an update. Trends Cogn Sci 8:539-546.

Boussaoud D, Wise SP (1993a) Primate frontal cortex: effects of stimulus and movement. Exp Brain Res 95:28-40.

Boussaoud D, Wise SP (1993b) Primate frontal cortex: neuronal activity following attentional versus intentional cues. Exp Brain Res 95:15-27.

Boynton GM, Engel SA, Glover GH, Heeger DJ (1996) Linear systems analysis of functional magnetic resonance imaging in human V1. J Neurosci 16:4207-4221.

Brass M, von Cramon DY (2002) The role of the frontal cortex in task preparation. Cereb Cortex 12:908-914.

Brass M, von Cramon DY (2004a) Decomposing components of task preparation with functional magnetic resonance imaging. J Cogn Neurosci 16:609-620.

Brass M, von Cramon DY (2004b) Selection for cognitive control: a functional magnetic resonance imaging study on the selection of task-relevant information. J Neurosci 24:8847-8852.

Brass M, Derrfuss J, Forstmann B, von Cramon DY (2005) The role of the inferior frontal junction area in cognitive control. Trends Cogn Sci 9:314-316.

Bunge SA (2005) How we use rules to select actions: a review of evidence from cognitive neuroscience. Cogn Affect Behav Neurosci 4:564-579.

Bunge SA, Klingberg T, Jacobsen RB, Gabrieli JD (2000) A resource model of the neural basis of executive working memory. Proc Natl Acad Sci USA 97:3573-3578.

Bunge SA, Hazeltine E, Scanlon MD, Rosen AC, Gabrieli JD (2002) Dissociable contributions of prefrontal and parietal cortices to response selection. NeuroImage 17:1562-1571.

Bunge SA, Kahn I, Wallis JD, Miller EK, Wagner AD (2003) Neural circuits subserving the retrieval and maintenance of abstract rules. J Neurophysiol 90:3419-3428.

Cisek P, Kalaska JF (2005) Neural correlates of reaching decisions in dorsal premotor cortex. Neuron 45:801-814.

Corbetta M, Shulman GL (2002) Control of goal-directed and stimulusdriven attention in the brain. Nat Rev Neurosci 3:201-215.

Crone EA, Wendelken C, Donohue SE, Bunge SA (2005) Neural evidence for dissociable components of task-switching. Cereb Cortex, in press.
Culham JC, Cavanagh P, Kanwisher NG (2001) Attention response functions: characterizing brain areas using $\mathrm{PMRI}$ activation during parametric variations of attentional load. Neuron 32:737-745.

Cunnington R, Windischberger C, Deecke L, Moser E (2003) The preparation and readiness for voluntary movement: a high-field event-related fMRI study of the Bereitschafts-BOLD response. NeuroImage 20:404-412.

Damasio H (1995) Human brain anatomy in computerized images. Oxford: Oxford UP.

Deiber MP, Ibanez V, Sadato N, Hallett M (1996) Cerebral structures participating in motor preparation in humans: a positron emission tomography study. J Neurophysiol 75:233-247.

Donders FC (1969) On the speed of mental processes. Acta Psychologica (Amst) 30:412-431.

Duvernoy HM (1999) The human brain: surface, three-dimensional sectional anatomy and MRI. Vienna: Springer.

Fox MD, Snyder AZ, Vincent JL, Corbetta M, Van Essen DC, Raichle ME (2005) From the cover: the human brain is intrinsically organized into dynamic, anticorrelated functional networks. Proc Natl Acad Sci USA 102:9673-9678.

Freire L, Mangin JF (2001) Motion correction algorithms may create spurious brain activations in the absence of subject motion. NeuroImage 14:709-722.

Greicius MD, Menon V (2004) Default-mode activity during a passive sensory task: uncoupled from deactivation but impacting activation. J Cogn Neurosci 16:1484-1492.

Greicius MD, Krasnow B, Reiss AL, Menon V (2003) Functional connectivity in the resting brain: a network analysis of the default mode hypothesis. Proc Natl Acad Sci USA 100:253-258.

Hazeltine E, Poldrack R, Gabrieli JD (2000) Neural activation during response competition. J Cogn Neurosci 12 [Suppl 2]:118-129.

Hazeltine E, Bunge SA, Scanlon MD, Gabrieli JD (2003) Materialdependent and material-independent selection processes in the frontal and parietal lobes: an event-related fMRI investigation of response competition. Neuropsychologia 41:1208-1217.

Huang MX, Harrington DL, Paulson KM, Weisend MP, Lee RR (2004) Temporal dynamics of ipsilateral and contralateral motor activity during voluntary finger movement. Hum Brain Mapp 23:26-39.

Jennings JR, van der Molen MW (2005) Preparation for speeded action as a psychophysiological concept. Psychol Bull 131:434-459.

Jiang Y, Kanwisher N (2003a) Common neural mechanisms for response selection and perceptual processing. J Cogn Neurosci 15:1095-1110.

Jiang Y, Kanwisher N (2003b) Common neural substrates for response selection across modalities and mapping paradigms. J Cogn Neurosci 15:1080-1094.

Kastner S, Pinsk MA, De Weerd P, Desimone R, Ungerleider LG (1999) Increased activity in human visual cortex during directed attention in the absence of visual stimulation. Neuron 22:751-761.

Kimberg DY, Aguirre GK, D’Esposito M (2000) Modulation of task-related neural activity in task-switching: an fMRI study. Brain Res Cogn Brain Res 10:189-196.

Klassen LM, Menon RS (2004) Robust automated shimming technique using arbitrary mapping acquisition parameters (RASTAMAP). Magn Reson Med 51:881-887.

Lee KM, Chang KH, Roh JK (1999) Subregions within the supplementary motor area activated at different stages of movement preparation and execution. NeuroImage 9:117-123.

Luks TL, Simpson GV, Feiwell RJ, Miller WL (2002) Evidence for anterior cingulate cortex involvement in monitoring preparatory attentional set. NeuroImage 17:792-802.

Ogawa S, Tank DW, Menon R, Ellermann JM, Kim SG, Merkle H, Ugurbil K (1992) Intrinsic signal changes accompanying sensory stimulation: functional brain mapping with magnetic resonance imaging. Proc Natl Acad Sci USA 89:5951-5955.

Passingham RE (1993) The frontal lobes and voluntary actions. New York: Oxford UP.

Pasupathy A, Miller EK (2005) Different time courses of learning-related activity in the prefrontal cortex and striatum. Nature 433:873-876.

Paus T (2001) Primate anterior cingulate cortex: where motor control, drive and cognition interface. Nat Rev Neurosci 2:417-424.

Petit L, Courtney SM, Ungerleider LG, Haxby JV (1998) Sustained activity 
in the medial wall during working memory delays. J Neurosci 18:9429-9437.

Picard N, Strick PL (2001) Imaging the premotor areas. Curr Opin Neurobiol 11:663-672.

Pochon JB, Levy R, Poline JB, Crozier S, Lehericy S, Pillon B, Deweer B, Le Bihan D, Dubois B (2001) The role of dorsolateral prefrontal cortex in the preparation of forthcoming actions: an fMRI study. Cereb Cortex 11:260-266.

Price CJ, Friston KJ (1997) Cognitive conjunction: a new approach to brain activation experiments. NeuroImage 5:261-270.

Raichle ME (2001) Cognitive neuroscience: bold insights. Nature 412:128-130.

Roland PE, Larsen B, Lassen NA, Skinhoj E (1980) Supplementary motor area and other cortical areas in organization of voluntary movements in man. J Neurophysiol 43:118-136.

Rushworth MF, Paus T, Sipila PK (2001) Attention systems and the organization of the human parietal cortex. J Neurosci 21:5262-5271.

Rushworth MF, Johansen-Berg H, Gobel SM, Devlin JT (2003) The left parietal and premotor cortices: motor attention and selection. NeuroImage 20 [Suppl 1]:S89-S100.

Rushworth MFS, Nixon PD, Passingham RE (1997) Parietal cortex and movement. Exp Brain Res 117:292-310.

Schluter ND, Rushworth MF, Passingham RE, Mills KR (1998) Temporary interference in human lateral premotor cortex suggests dominance for the selection of movements. A study using transcranial magnetic stimulation. Brain 121:785-799.

Schluter ND, Rushworth MF, Mills KR, Passingham RE (1999) Signal-, set-, and movement-related activity in the human premotor cortex. Neuropsychologia 37:233-243.

Schumacher EH, Elston PA, D'Esposito M (2003) Neural evidence for representation-specific response selection. J Cogn Neurosci 15:1111-1121.

Shulman GL, d'Avossa G, Tansy AP, Corbetta M (2002) Two attentional processes in the parietal lobe. Cereb Cortex 12:1124-1131.
Smith EE, Jonides J (1997) Working memory: a view from neuroimaging. Cognit Psychol 33:5-42.

Sohn MH, Ursu S, Anderson JR, Stenger VA, Carter CS (2000) Inaugural article: the role of prefrontal cortex and posterior parietal cortex in task switching. Proc Natl Acad Sci USA 97:13448-13453.

Stoet G, Snyder LH (2004) Single neurons in posterior parietal cortex of monkeys encode cognitive set. Neuron 42:1003-1012.

Talairach J, Tournoux P (1988) Co-planar stereotaxic atlas of the human brain. New York: Thieme Medical Publishers.

Tanji J (1996) New concepts of the supplementary motor area. Curr Opin Neurobiol 6:782-787.

Thickbroom GW, Byrnes ML, Sacco P, Ghosh S, Morris IT, Mastaglia FL (2000) The role of the supplementary motor area in externally timed movement: the influence of predictability of movement timing. Brain Res 874:233-241.

Thoenissen D, Zilles K, Toni I (2002) Differential involvement of parietal and precentral regions in movement preparation and motor intention. J Neurosci 22:9024-9034.

Toni I, Schluter ND, Josephs O, Friston K, Passingham RE (1999) Signal-, set- and movement-related activity in the human brain: an event-related fMRI study. Cereb Cortex 9:35-49.

Toni I, Thoenissen D, Zilles K (2001) Movement preparation and motor intention. NeuroImage 14:S110-S117.

Wise SP (1993) Monkey motor cortex: movements, muscles, motoneurons and metrics. Trends Neurosci 16:46-49.

Wise SP, Di Pellegrino G, Boussaoud D (1992) Primate premotor cortex: dissociation of visuomotor from sensory signals. J Neurophysiol 68:969-972.

Wojciulik E, Kanwisher N (1999) The generality of parietal involvement in visual attention. Neuron 23:747-764.

Wojciulik E, Kanwisher N, Driver J (1998) Covert visual attention modulates face-specific activity in the human fusiform gyrus: fMRI study. J Neurophysiol 79:1574-1578. 